\title{
Preparation of bufalin-loaded pluronic polyetherimide nanoparticles, cellular uptake, distribution, and effect on colorectal cancer
}

This article was published in the following Dove Press journal:

International Journal of Nanomedicine

2I August 2014

Number of times this article has been viewed

\author{
Qiang $\mathrm{Hu}^{1,3, *}$ \\ Bo Liang, 2,* \\ Ying Sun ${ }^{4}$ \\ Xiao-Ling Guo, \\ Yi-Jie $\mathrm{Bao}^{2}$ \\ Dong-Hao $\mathrm{Xie}^{3}$ \\ Ming Zhou ${ }^{3}$ \\ You-Rong Duan ${ }^{4}$ \\ Pei-Hao Yin ${ }^{2}$ \\ Zhi-Hai Peng' \\ 'Department of Hepatobiliary Surgery, \\ Qianfoshan Hospital, Shandong \\ University, Jinan, ${ }^{2}$ Department \\ of General Surgery, Putuo Hospital, \\ Shanghai University of Traditional \\ Chinese Medicine, ${ }^{3}$ Department \\ of General Surgery, Dahua Hospital, \\ ${ }^{4}$ State Key Laboratory of Oncogenes \\ and Related Genes, Shanghai Cancer \\ Institute, Renji Hospital, School \\ of Medicine, Shanghai Jiao Tong \\ University, Shanghai, People's Republic \\ of China \\ *These authors contributed equally \\ to this work
}

Correspondence: Pei-Hao Yin Department of General Surgery, Putuo Hospital, Shanghai University of Traditional Chinese Medicine, I 64 Lanxi Rd, Shanghai 200062, People's Republic of China

$\mathrm{Tel}+862$ II 22233836

Fax +86 2I 52665957

Email yinpeihao1975@hotmail.com

Zhi-Hai Peng

Department of Hepatobiliary Surgery,

Qianfoshan Hospital, Shandong

University, Jinan, 2500 I4, People's

Republic of China

Tel +862I 63240090 ext 3I32

Email zhihai.peng@hotmail.com
Abstract: A large number of studies have shown that bufalin can have a significant antitumor effect in a variety of tumors. However, because of toxicity, insolubility in water, fast metabolism, short half-life, and other shortcomings, its application is limited in cancer therapy. In this study, we explored the anti-metastatic role of bufalin-loaded pluronic polyetherimide nanoparticles on HCT116 colon cancer-bearing mice. Nanoparticle size, shape, drug loading, encapsulation efficiency, and in vitro drug release were studied. Also, cellular uptake of nanoparticles, in vivo tumor targeting, and tumor metastasis were studied. The nanoparticles had a particle size of about $60 \mathrm{~nm}$ and an encapsulation efficiency of $75.71 \%$, by weight. The in vitro release data showed that free bufalin was released faster than bufalin-loaded pluronic polyetherimide nanoparticles, and almost $80 \%$ of free bufalin was released after 32 hours. Nanoparticles had an even size distribution, were stable, and had a slow release and a tumor-targeting effect. Bufalin-loaded pluronic polyetherimide nanoparticles can significantly inhibit the growth and metastasis of colorectal cancer.

Keywords: colon cancer, nanoparticles, tumor target, bufalin

\section{Introduction}

Colorectal cancer is the third most commonly diagnosed cancer in males and the second in females worldwide. ${ }^{1}$ The survival and prognosis of colorectal cancer patients is dependent on the stage of the tumor at the time of detection. Unfortunately, about $50 \%$ of patients display regional or distant metastases at the time of diagnosis. ${ }^{2}$ Therefore, the treatment of colon cancer has become a focus of research worldwide. The formation of metastasis is an enormously complex process, whereby tumor cells disseminate from the primary tumor to colonize distant organs. This multi-step process involves initial malignant cell invasion of the microenvironment, filtration into the bloodstream, survival during migration, and extravasation into distant organs. Subsequent steps, including proliferation, induction of angiogenesis, and evasion of apoptotic death, ${ }^{3}$ must be overcome by the malignant cell for successful metastasis. ${ }^{4}$ Although tumor invasion and metastasis is fatal, the molecular mechanism is not yet fully clear. Therefore, we are now looking for new treatments.

Bufalin is a soluble digoxin-like immunoreactive component of Chansu (a traditional Chinese medicine), and is an extract of dried toad venom from the skin and parotid venom glands of Bufo gargarizans or B. melanostictus,,-8 and can produce a significant antitumor effect in a variety of tumors, including hepatocellular carcinoma, ${ }^{9}$ colorectal cancer, ${ }^{10}$ leukemia, ${ }^{11}$ and gastric cancer. ${ }^{12} \mathrm{~A}$ large number of studies have shown that antitumor effects of bufalin include inhibition of cell proliferation and 
induction of apoptosis of tumor cells. ${ }^{13-15}$ Because of its toxicity, insolubility in water, fast metabolism, short half-life, and other shortcomings, ${ }^{16}$ its application in cancer therapy is limited. However, studies have shown that nanocomposites can combine bufalin to overcome these shortcomings. ${ }^{17}$

Nanoparticulate drug delivery systems are making a significant contribution to the improvement of drug delivery in cancer. ${ }^{18,19}$ Targeting nanoparticulate drug delivery systems, especially biodegradable nanoparticles, provides opportunities to meet these existing challenges, with the advantages of improved pharmacokinetics, favored tumor accumulation, and reduced side effects. ${ }^{20-22}$ Hydrophilic pluronic polymers have a novel type of amphiphilic, nonionic surfactant micelle structure that is insoluble in the aqueous solution of the compound, which provides good solubility in the hydrophobic microenvironment, thereby increasing the solubility of water-soluble substances. The potential use of pluronic polymers as biomedical drug carriers, for controlled drug release and gene therapy, has been given much attention. Polyetherimide (PEI) is a class of cationic polymer with a large group structure, which increases the stability of pluronic polymeric composites.

In this study, we undertook to examine the role of bufalin-loaded pluronic PEI nanoparticles in colorectal cancer metastasis.

\section{Materials and methods Preparation of bufalin-loaded pluronic PEI nanoparticles}

Bufalin-loaded nanoparticles with pluronic PEI were prepared using a thin film method. Briefly, bufalin $(0.4 \mathrm{mg})$ and empty pluronic PEI nanoparticles (30 mg) were dissolved in methanol ( $4 \mathrm{~mL})$ and the organic solvent was subsequently removed by rotary vacuum evaporation at $30^{\circ} \mathrm{C}$, for a few minutes, to obtain a solid bufalin/copolymer matrix. Dissolution of the solid bufalin/copolymer matrix was carried out by adding water $(2 \mathrm{~mL})$ to the matrix and vigorously vortexing for a few minutes. Then, the solution was centrifuged at $3,000 \mathrm{rpm}$ for 15 minutes, to remove the drug crystals. The supernatant was the bufalin-loaded nanoparticles.

\section{Characterization of bufalin-loaded pluronic PEI nanoparticles}

Transmission electron microscopy (TEM) (Hitachi H-800; Hitachi Ltd, Tokyo, Japan) was used to study the morphology of the bufalin-loaded nanoparticles. The particle size distribution of the bufalin-loaded nanoparticles was determined using a Zetasizer Nano ZS (Malvern Instruments, Malvern, UK).

\section{Determination of drug loading}

parameters

The amount of bufalin in pluronic PEI nanoparticles was determined as follows. Briefly, the prepared bufalinloaded nanoparticles $(100 \mu \mathrm{L})$ were dissolved in $10 \mathrm{~mL}$ of methanol. The exact concentration of bufalin in the methanol solution was then determined by using ultraviolet-visible spectroscopy (TU-1901), for which the absorbance of bufalin at $298 \mathrm{~nm}$ was measured. Bufalin encapsulation efficiency (EE) and loading efficiency (LE) were calculated as follows:

$$
\begin{aligned}
& \text { EE } \%=(\text { weight drug loaded } / \\
&\text { weight drug input }) \times 100 \\
& \text { LE \% }=(\text { weight drug loaded } / \\
&\text { weight drug-loaded nanoparticles }) \times 100
\end{aligned}
$$

\section{In vitro release of bufalin from pluronic PEI nanoparticles}

The in vitro release properties of bufalin from pluronic PEI nanoparticles were investigated in an aqueous medium containing phosphate buffer saline (PBS) $(\mathrm{pH}=7.4)$, using a dialysis method. Bufalin-loaded nanoparticles were dispersed in $2 \mathrm{~mL}$ of PBS solution $(0.1 \mathrm{~mol} / \mathrm{L})$ and placed into a dialysis bag, with a molecular weight cut-off of 7,500 Da. Then, the sealed dialysis bag was immersed into $18 \mathrm{~mL}$ of the same PBS at $37^{\circ} \mathrm{C}$. The release medium was stirred at a speed of $80 \mathrm{rpm}$ for 32 hours. Samples were withdrawn at different time intervals (20 minutes, 40 minutes, 1, 2, 4, 6, 8, 10, 20, 24 , and 32 hours) and replaced with equal volumes of fresh PBS. The concentration of bufalin in the samples was determined by using ultraviolet-visible spectroscopy.

\section{Reagents}

Bufalin was purchased from Sigma-Aldrich (St Louis, MO, USA), dissolved in dimethyl sulfoxide (DMSO), as a vehicle, and stored in aliquots at $4{ }^{\circ} \mathrm{C}$. Roswell Park Memorial Institute 1,640 medium (RPMI) and fetal bovine serum (FBS) were purchased from Thermo Fisher Scientific (Waltham, MA, USA).

\section{Cell culture}

The human colorectal cancer cell line used was HCT116, purchased from the Cell Bank of Type Culture Collection of Chinese Academy of Sciences (Shanghai, People's Republic of China) and cultured in RPMI, supplemented with $10 \%$ FBS, penicillin (100 units $/ \mathrm{mL}$ ), and streptomycin (Thermo Fisher Scientific) $(100 \mathrm{~g} / \mathrm{mL})$ at $37^{\circ} \mathrm{C}$, in a $5 \% \mathrm{CO}_{2}$ humidified atmosphere. 


\section{Cellular uptake}

The cellular uptake experiments were performed using confocal laser scanning microscopy (CLSM) (Olympus FV-1000; Olympus Corporation, Tokyo, Japan). Rhodamine $\mathrm{B}(\mathrm{RB})$ was encapsulated in nanoparticles as a probe for the uptake study. HCT116 cells were seeded in 6-well plates at a density of $8 \times 10^{4}$ cells $/$ well and cultured for 24 hours at $37^{\circ} \mathrm{C}$ in $5 \% \mathrm{CO}_{2}$, followed by removal of the culture medium and addition of $500 \mathrm{~mL}$ of fresh medium, containing RB-loaded pluronic PEI nanoparticles and free $\mathrm{RB}$, at the designated RB concentration of $20 \mu \mathrm{g} / \mathrm{mL}$. After incubation for 1 hour, the culture medium was removed and the cells were washed with PBS three times. The cells were then fixed with $4 \%$ formaldehyde for 30 minutes at room temperature, and the slides were rinsed with PBS three times. Finally, the cells were stained with 4,6-diamidino-2-phenylindole (DAPI) for 10 minutes, then rinsed with PBS three times. The slides were mounted and examined via CLSM.

\section{In vivo metastasis assay}

Male athymic nude mice were randomly divided into five groups: (A) HCT116-D-luciferin-[luc-] control, (B) HCT116luc-control + blank pluronic PEI nanoparticles, (C) HCT116-luc-control + bufalin, (D) HCT116-luc-control + bufalin-loaded pluronic PEI nanoparticles, (E) HCT116-luccontrol + oxaliplatin (L-ohp) (ten mice per group). A cluster of $1 \times 10^{6}$ cells was injected intravenously, via the tail vein. After 2 weeks, group B was treated with $19 \mathrm{mg} / \mathrm{kg}$ of blank PEI nanoparticles, group C was treated with $1 \mathrm{mg} / \mathrm{kg}$ of bufalin, group D was treated with $20 \mathrm{mg} / \mathrm{kg}$ of bufalin-loaded pluronic PEI nanoparticles, and group E was treated with $5 \mathrm{mg} / \mathrm{kg}$ of L-ohp in $0.9 \%$ physiological saline, via the tail vein $(0.2 \mathrm{~mL}$ per mouse, three times per week, for 3 weeks). The control (group A) received the same isovolumetric dose of $0.9 \%$ physiological saline, by tail vein injection.

Tumor growth and metastasis were analyzed using in vivo optical imaging. All mice were euthanized 3 weeks after initial injection. Livers were excised and embedded in paraffin for section and staining with hematoxylin-eosin (H\&E).

\section{In vivo optical imaging}

Prior to in vivo imaging, the mice were anesthetized with phenobarbital sodium. D-luciferin solution $(150 \mathrm{mg} / \mathrm{kg})$ was injected, intraperitoneally, 5 minutes before imaging. The exposure time of bioluminescence imaging ranged from 10-30 seconds. Fluorescence imaging was performed with an excitation wavelength of $490 \mathrm{~nm}$ and emission wavelength of $535 \mathrm{~nm}$. The exposure time ranged from 1-2 minutes.

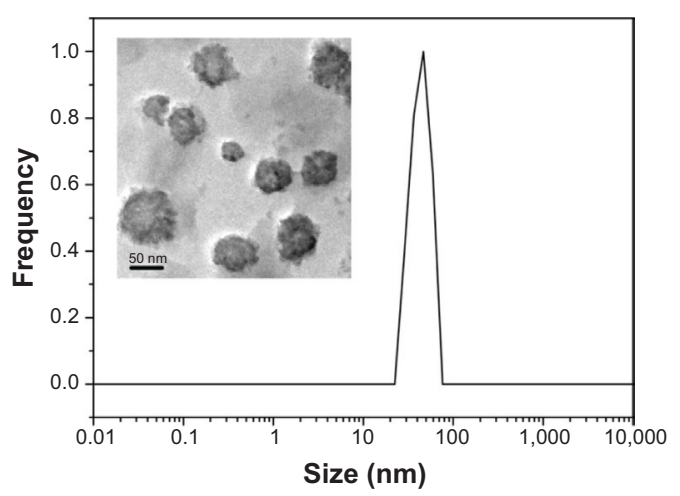

Figure I Size distribution and TEM micrograph of bufalin-loaded pluronic PEI nanoparticles.

Abbreviations: TEM, transmission electron microscopy; PEI, polyetherimide.

\section{Results}

Figure 1 shows the size distribution and TEM micrograph of bufalin-loaded pluronic PEI nanoparticles. Bufalin-loaded pluronic PEI nanoparticles showed a narrow size distribution, ranging from 30-80 $\mathrm{nm}$, with an average particle diameter of $65 \mathrm{~nm}$ (polydispersity index [PDI] $=0.214$ ). The size and morphology of bufalin-loaded pluronic PEI nanoparticles were further evaluated by TEM. As shown in the inset, the bufalin-loaded pluronic PEI nanoparticles, with an average diameter of about $60 \mathrm{~nm}$, are well-shaped spheres. The zeta potential of bufalin-loaded pluronic PEI nanoparticles was $5.87 \pm 0.42 \mathrm{mV}$.

The bufalin-encapsulating efficiency of the pluronic PEI nanoparticles was $75.71 \%$, by weight, whereas the loading efficiency was $3.04 \%$, by weight. The in vitro release profile of bufalin-loaded pluronic PEI nanoparticles, obtained at $37^{\circ} \mathrm{C}$, can be seen in Figure 2. The release of free bufalin was also investigated, as a control. In neutral surroundings

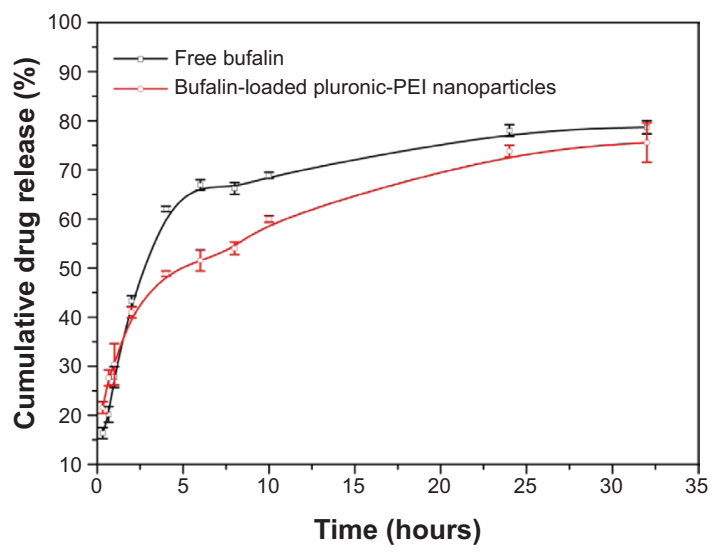

Figure 2 Drug release profiles of free bufalin and bufalin-loaded pluronic PEI nanoparticles at $37^{\circ} \mathrm{C}$.

Note: Data is shown as mean \pm standard deviation; $n=3$.

Abbreviation: PEl, polyetherimide. 


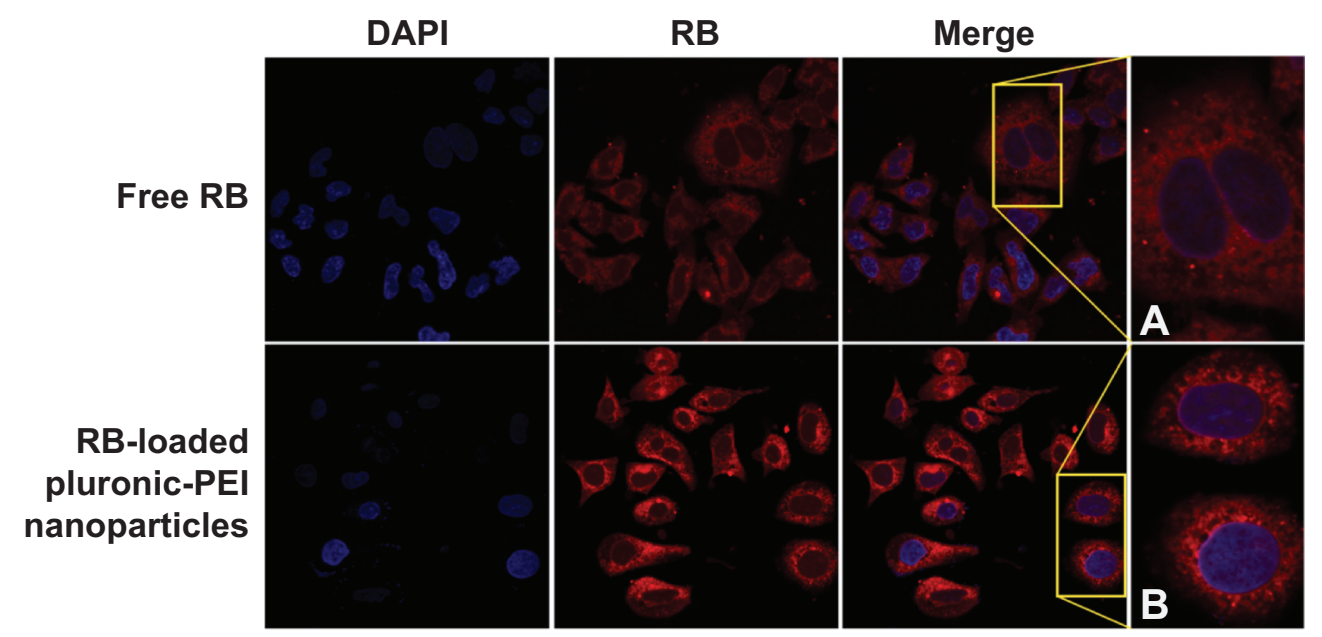

Figure 3 Confocal laser scanning microscopy images of HCTI 16 cells cultured with free RB and RB-loaded pluronic PEI nanoparticles. Notes: The concentration of RB was $20 \mathrm{mg} / \mathrm{mL}$, for 4 hours. (A) and (B) are magnified images.

Abbreviations: RB, rhodamine B; PEI, polyetherimide; DAPI, 4,6-diamidino-2-phenylindole.

(pH 7.4), the release rates were apparently different under identical conditions. This may be attributable to different hydrophobic and ionic interaction between bufalin and pluronic PEI nanoparticles. In vitro release data showed that free bufalin was released faster than bufalin-loaded pluronic PEI nanoparticles; almost $80 \%$ of free bufalin was released after 32 hours. It can be concluded that the release rate of the drug will largely be a function of its localization within the micelle. The outer corona region of the micelle is quite mobile. As a result, release from this area will be rapid. The release of drug localized in the corona, or at the interface, is believed to account for "burst release" from the micelles.

To verify the effect of cellular uptake of pluronic PEI nanoparticles, a CLSM study was conducted. We took advantage of the red autofluorescence from RB and blue fluorescence from DAPI to study the cellular uptake of the nanoparticles. The cells were incubated with the RB-loaded pluronic PEI nanoparticles, and free RB, for 4 hours. Then, the cell nuclei were stained with DAPI. It can be seen in Figure 3 that, after 4 hours of exposure, the HCT116
A

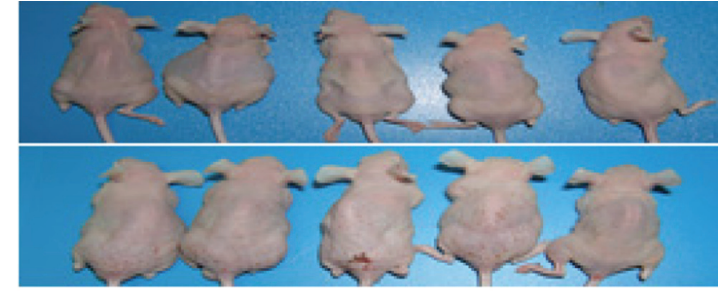

C

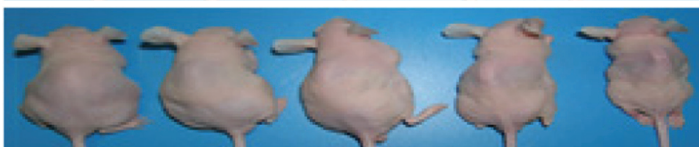

D

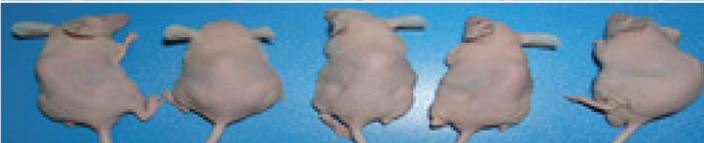

E

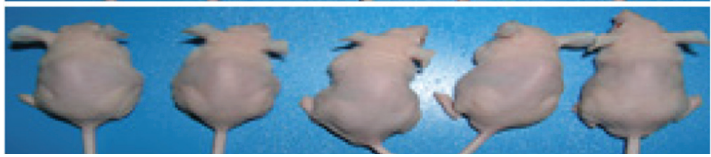

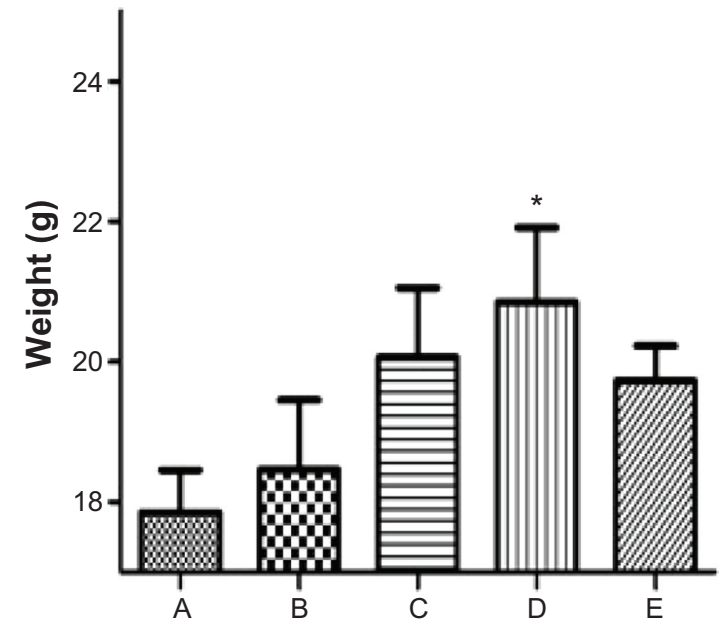

The weight of nude mice in different groups $(n=10)$

Figure 4 Images showing the general condition, and the weight of the mice in groups A-E at 3 weeks after the initial injection.

Notes: The error bars show standard deviation; $F=19.864 ; P<0.005$. Statistical significance was defined as follows: $* P<0.047$ versus group $C$. There were demonstrably different physiological characteristics in group $D$ in terms of body weight and skin roughness, compared with groups $A, B, C$, and $E$.

Abbreviation: PEl, polyetherimide. 
cells treated with free RB remained largely blue; little red appeared, indicating that the intracellular presence of $\mathrm{RB}$ was small and random. RB-loaded pluronic PEI nanoparticles exhibited high fluorescence intensity in HCT116, compared with free RB. It was found that RB-loaded pluronic PEI nanoparticles enhanced cellular uptake efficiency in HCT116 cells.

There were significantly improved physiological characteristics in groups C (HCT116-luc-control + bufalin), D (HCT116-luc-control + bufalin-loaded pluronic PEI nanocapsules), and E (HCT116-luc-control + L-ohp), in terms of body weight, skin roughness, and mental status, compared with groups A (HCT116-luc-control) and B (HCT116-luccontrol + blank pluronic PEI nanocapsules). These results indicated that bufalin, bufalin-loaded pluronic PEI nanocapsules, and L-ohp inhibited colorectal cancer metastasis and improved quality of life. It is of particular note that these results were more pronounced in group D (Figure 4).

In vivo optical imaging revealed successively decreased metastatic lesion size and number in groups $\mathrm{C}, \mathrm{D}$, and $\mathrm{E}$, compared with groups A and B, 3 weeks after bufalin treatment, indicating that bufalin-loaded pluronic PEI nanocapsules, bufalin, and L-ohp markedly inhibited colorectal cancer metastasis. Bufalin-loaded pluronic PEI nanocapsules showed a stronger inhibitory effect than bufalin administration alone (Figure 5).

The images of livers and H\&E staining in each group, 3 weeks after treatment, indicated decreased metastatic lesion size and number in groups $\mathrm{C}, \mathrm{D}$, and $\mathrm{E}$, compared with groups A and B, 3 weeks after treatment. Markedly inhibited colorectal cancer metastasis was shown in groups $\mathrm{C}, \mathrm{D}$, and E. The inhibitory effect on colorectal cancer metastasis of bufalin-loaded pluronic PEI nanocapsules exceeded that of bufalin administration alone. H\&E staining gave similar results; liver structure damage from metastasis was progressively lower in groups $\mathrm{C}, \mathrm{D}$, and $\mathrm{E}$, compared with groups $\mathrm{A}$ and $\mathrm{B}$. As shown, metastatic damage in groups $\mathrm{A}$ and $\mathrm{B}$ was more serious and more complete (Figure 6).

\section{Discussion}

Bufalin's side effects, especially its toxicity, are a serious impediment to its use in clinical treatment. However, nanoparticles can provide bufalin to the tumor site accurately, thereby reducing its toxicity to normal tissue.

Nanomaterials are important materials, due to their unique physical and chemical properties. ${ }^{23}$ Due to the accumulation of nanoparticles within the cell, through their enhanced permeability and retention effects, nanoparticles achieve

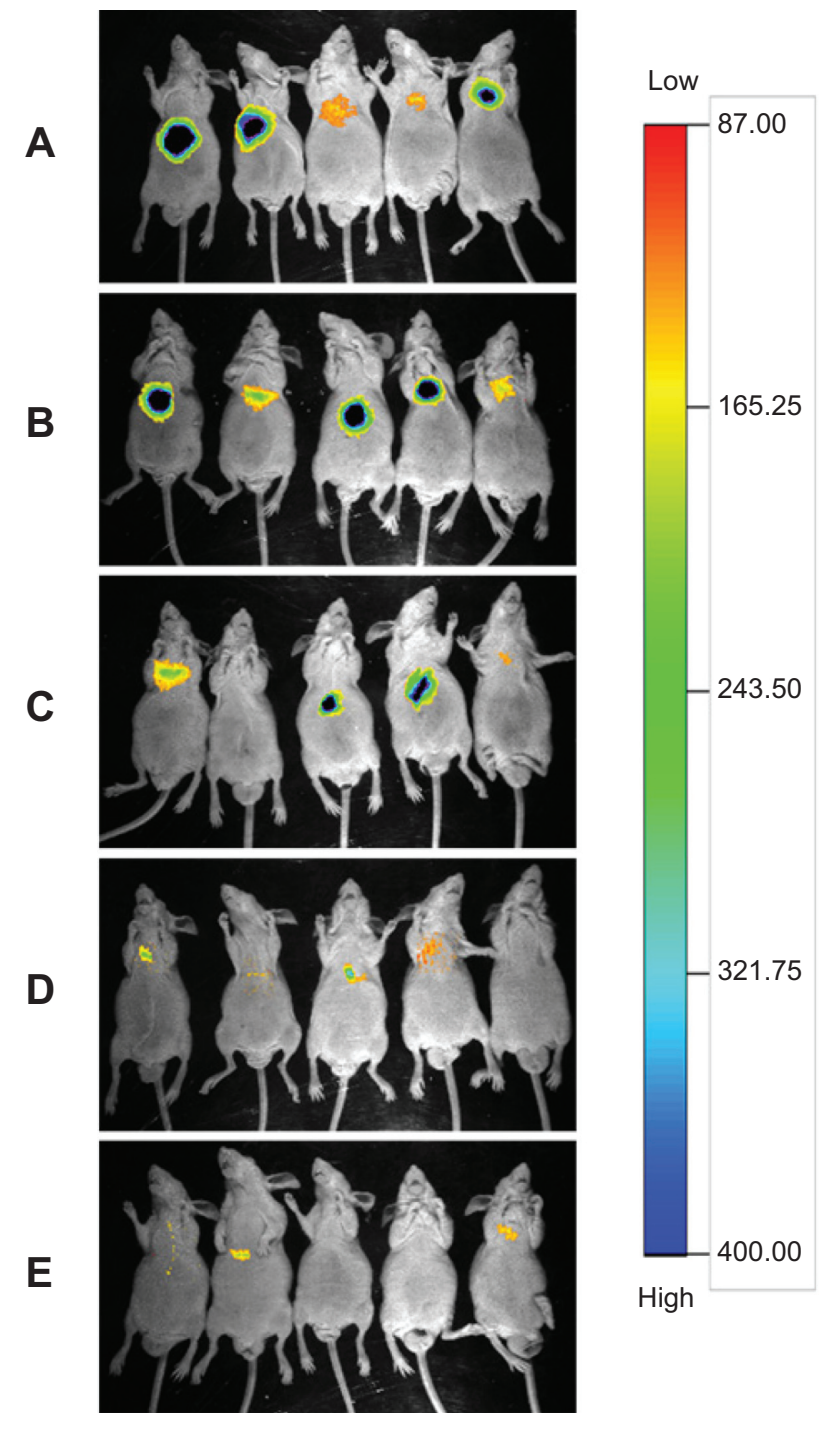

Figure $\mathbf{5}$ In vivo noninvasive targeted imaging of tumor-bearing mice injected with (A) physiological saline, (B) pluronic PEl blank nanocapsules, (C) bufalin, (D) bufalinloaded pluronic PEI nanocapsules, and (E) L-ohp. The colors indicate the changes in fluorescence signal intensity, from low (red) to high (blue).

Notes: In vivo optical imaging revealed successively decreased metastatic lesion size and number in groups C, D, and E, compared with groups A and B, 3 weeks after bufalin treatment, indicating that bufalin-loaded pluronic PEI nanocapsules, bufalin, and L-ohp markedly inhibited colorectal cancer metastasis. Bufalin-loaded pluronic PEI nanocapsules showed stronger inhibitory effects than bufalin administration alone.

Abbreviations: PEl, polyetherimide; L-ohp, oxaliplatin.

new biological properties. ${ }^{24,25}$ Because nanoformulations can reduce the potential for some specific antineoplastic systemic toxicities, and other side effects, it is a drug delivery system that has good prospects for development. Studies have shown that tumor cells have a strong endothelial cell gap and phagocytic lesions. The tumor-endothelial cell gap is much larger than normal, and is therefore more accessible to nanoparticles within the tumor, while prolonged sustained antineoplastic drug residence time extends the duration of action and improves efficacy. ${ }^{26}$ 
A
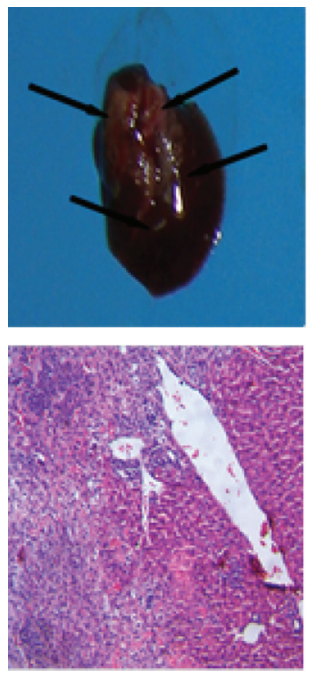

B
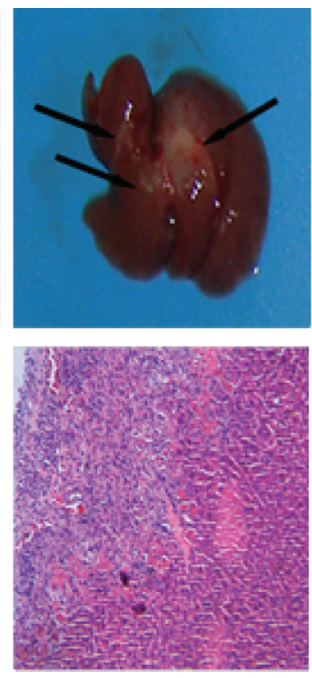

C
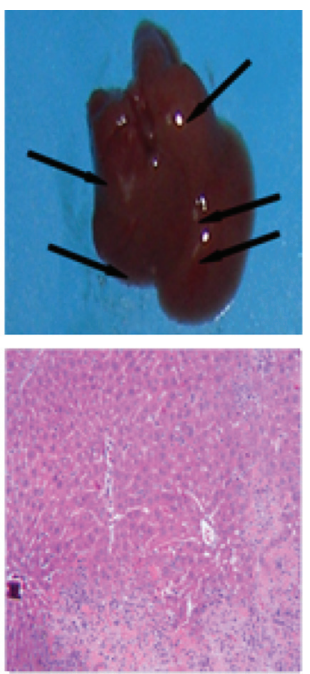

D
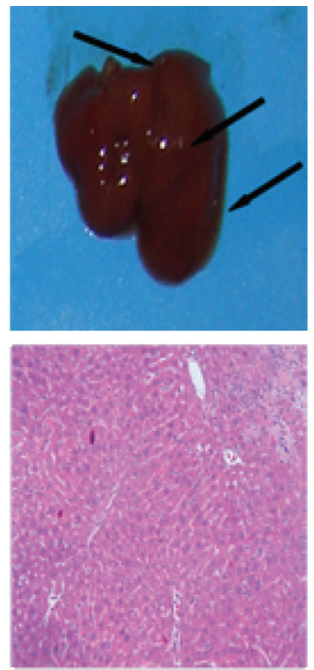

E
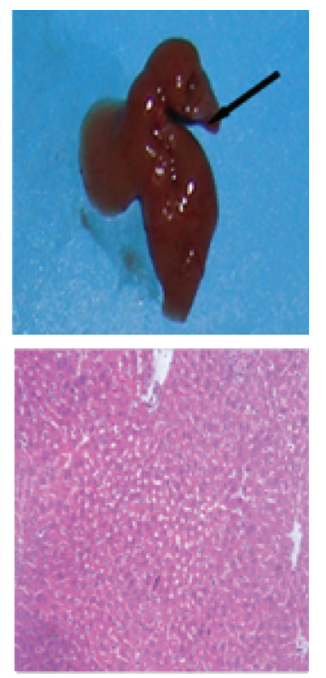

Figure 6 Images of livers (upper images) and H\&E staining in each group (lower images), 3 weeks after treatment.

Notes: The images of livers in each group indicated decreased metastatic lesion size and number in groups C, D, and E, compared with groups A and B, 3 weeks after treatment. Markedly inhibited colorectal cancer metastasis was shown in groups C, D, and E. The inhibitory effect on colorectal cancer metastasis of bufalin-loaded pluronic PEI nanocapsules exceeded that of bufalin administration alone. H\&E staining gave similar results; liver structure damage caused by metastasis was lower in groups $C$, D, and $E$ than in groups $A$ and $B$. As shown, metastatic damage in groups A and B was more serious and more complete.

Abbreviations: PEl, polyetherimide; H\&E, hematoxylin-eosin.

In this study, we successfully prepared bufalin-loaded pluronic PEI nanoparticles of the desired size $(60 \mathrm{~nm})$, for treatment of colorectal cancer. Our experimental results showed that the bufalin-loaded pluronic PEI nanoparticles had a particle size of about $60 \mathrm{~nm}$ and an encapsulation efficiency of $75.71 \%$, by weight. In vitro release data showed that free bufalin was released faster than in bufalin-loaded pluronic PEI nanoparticles; almost $80 \%$ of free bufalin was released after 32 hours. It was found that RB-loaded pluronic PEI nanoparticles enhanced cellular uptake efficiency in HCT116 cells. For the bufalin-loaded pluronic PEI nanoparticles prepared in this experiment, in vitro cellular uptake experiments and in vivo fluorescence imaging experiments have successfully demonstrated that the nanoparticles can effectively target tumors. These results indicate that bufalin-loaded pluronic PEI nanoparticles have the ability to enhance therapeutic effect such as the inhibition of tumor growth and metastasis. The evidence shows a significantly enhanced potential of nanoparticles to deliver anticancer drugs, to enhance antitumor effects.

Our results showed that nanocarriers based on pluronic PEI nanomaterials with controlled release action protected normal tissues against harm from bufalin during blood circulation. They also achieved targeted, controlled release. The results showed that bufalin-loaded nanoparticles, compared with the same dose of bufalin, more obviously inhibited tumor growth and metastasis of colon cancer in nude mice, improving their quality of life.
These data indicate that the nanoparticles can help to improve colorectal cancer therapy. However, this study is mainly focused on the current delivery capacity of nanoparticles to cells, and on in vivo imaging. Further in-depth study of the nanoparticles will be needed in colorectal cancer therapy.

\section{Acknowledgments}

This project was supported by the Shanghai Committee of Science and Technology, China (Number 114119b3100), and by the Putuo District Committee of Science and Technology, Shanghai, China (Number 201102). This research work was also supported by Construct Program of the Key Discipline of State Administration of Traditional Chinese Medicine of People's Republic of China. The four affiliations contributed equally to this work, thanks for the support of the four units.

\section{Disclosure}

The authors report no conflicts of interest in this work.

\section{References}

1. Jemal A, Bray F, Center MM, Ferlay J, Ward E, Forman D. Global cancer statistics. CA Cancer J Clin. 2011;61(2):69-90.

2. Figueredo A, Coombes ME, Mukherjee S. Adjuvant therapy for completely resected stage II colon cancer. Cochrane Database Syst Rev. 2008;16(3):CD005390.

3. Hunter KW, Crawford NP, Alsarraj J. Mechanisms of metastasis. Breast Cancer Res. 2008;10 Suppl 1:S2.

4. Dykxhoorn DM. MicroRNAs and metastasis: little RNAs go a long way. Cancer Res. 2010;70(16):6401-6406.

5. Krenn L, Kopp B. Bufadienolides from animal and plant sources. Phytochemistry. 1998;48(1):1-29. 
6. Qi F, Li A, Inagaki Y, et al. Antitumor activity of extracts and compounds from the skin of the toad Bufo bufo gargarizans Cantor. Int Immunopharmacol. 2011;11(3):342-349.

7. Lu CX, Nan KJ, Lei Y. Agents from amphibians with anticancer properties. Anticancer Drugs. 2008;19(10):931-939.

8. Cui X, Inagaki Y, Xu H, et al. Anti-hepatitis B virus activities of cinobufacini and its active components bufalin and cinobufagin in HepG2.2.15 cells. Biol Pharm Bull. 2010;33(10):1728-1732.

9. Miao Q, Bi LL, Li X, et al. Anticancer effects of Bufalin on human hepatocellular carcinoma HepG2 Cells: roles of apoptosis and autophagy. Int J Mol Sci. 2013;14(1):1370-1382.

10. Xie CM, Chan WY, Yu S, Zhao J, Cheng CH. Bufalin induces autophagy-mediated cell death in human colon cancer cells through reactive oxygen species generation and JNK activation. Free Radic Biol Med. 2011;51(7):1365-1375.

11. Amano Y, Cho Y, Matsunawa M, Komiyama K, Makishima M. Increased nuclear expression and transactivation of vitamin $\mathrm{D}$ receptor by the cardiotonic steroid bufalin in human myeloid leukemia cells. J Steroid Biochem Mol Biol. 2009;114(3-5):144-151.

12. Li D, Qu X, Hou K, et al. PI3K/Akt is involved in bufalin-induced apoptosis in gastric cancer cells. Anticancer Drugs. 2009;20(1):59-64

13. Shen S, Zhang Y, Wang Z, Liu R, Gong X. Bufalin induces the interplay between apoptosis and autophagy in glioma cells through endoplasmic reticulum stress. Int J Biol Sci. 2014;10(2):212-224.

14. Tsai SC, Yang JS, Peng SF, et al. Bufalin increases sensitivity to AKT/ mTOR-induced autophagic cell death in SK-HEP-1 human hepatocellular carcinoma cells. Int J Oncol. 2012;41(4):1431-1442.

15. Chen YY, Lu HF, Hsu SC, et al. Bufalin inhibits migration and invasion in human hepatocellular carcinoma SK-Hep1 cells through the inhibitions of NF-kB and matrix metalloproteinase-2/-9-signaling pathways. Environ Toxicol. 2013, Aug 16.

16. Gong LL, Li JX, Zhang HM. Progress on pharmacological action and preparation of Venenum Bufonis. Food Drug. 2007;9(10):51-53.
17. Yin $P$, Wang Y, Qiu Y, et al. Bufalin-loaded mPEG-PLGA-PLL-cRGD nanoparticles: preparation, cellular uptake, tissue distribution, and anticancer activity. Int J Nanomedicine. 2012;7:3961-3969.

18. Kim SS, Rait A1, Rubab F, et al. The clinical potential of targeted Nanomedicine: delivering to cancer stem-like cells. Mol Ther. 2014; 22(2):278-291.

19. Xia H, Gao X, Gu G, et al. Low molecular weight protamine-functionalized nanoparticles for drug delivery to the brain after intranasal administration. Biomaterials. 2011;32(36):9888-9898.

20. Ferrari M. Cancer nanotechnology: opportunities and challenges. Nat Rev Cancer. 2005;5(3):161-171.

21. Davis ME, Chen ZG, Shin DM. Nanoparticle therapeutics: an emerging treatment modality for cancer. Nat Rev Drug Discov. 2008; 7(9):771-782.

22. Patel NR, Chen ZG, Shin DM. Nanopreparations to overcome multidrug resistance in cancer. Adv Drug Deliv Rev. 2013;65(13-14): 1748-1762.

23. Venkatasubbu GD, Ramasamy S, Avadhani GS, Palanikumar L, Kumar J. Size-mediated cytotoxicity of nanocrystalline titanium dioxide, pure and zinc-doped hydroxyapatite nanoparticles in human hepatoma cells. J Nanopart Res. 2012;14:819-836.

24. Kasemets K, Ivask A, Dubourguier HC, Kahru A. Toxicity of nanoparticles of $\mathrm{ZnO}, \mathrm{CuO}$ and $\mathrm{TiO} 2$ to yeast Saccharomyces cerevisiae. Toxicol in Vitro. 2009;23(6):1116-1122.

25. Lee BS, Park K, Park S, et al. Tumor targeting efficiency of bare nanoparticles does not mean the efficacy of loaded anticancer drugs: importance of radionuclide imaging for optimization of highly selective tumor targeting polymeric nanoparticles with or without drug. J Control Release. 2010;147(2):253-260.

26. Li F, Wang Y, Liu Z, Lin X, He H, Tang X. Formulation and characterization of bufadienolides-loaded nanostructured lipid carriers. Drug Dev Ind Pharm. 2010;36(5):508-517.
International Journal of Nanomedicine

\section{Publish your work in this journal}

The International Journal of Nanomedicine is an international, peerreviewed journal focusing on the application of nanotechnology in diagnostics, therapeutics, and drug delivery systems throughout the biomedical field. This journal is indexed on PubMed Central, MedLine, CAS, SciSearch $®$, Current Contents $\AA /$ Clinical Medicine,

\section{Dovepress}

Journal Citation Reports/Science Edition, EMBase, Scopus and the Elsevier Bibliographic databases. The manuscript management system is completely online and includes a very quick and fair peer-review system, which is all easy to use. Visit http://www.dovepress.com/ testimonials.php to read real quotes from published authors. 